\title{
El modelo UPPS de impulsividad en el abuso de las \\ Tecnologías de la Información y la Comunicación (TIC)
}

\section{The UPPS model of impulsivity in the abuse of Information and Communication Technologies (ICT)}

\author{
Eduardo J. Pedrero Pérez*, Sara Morales Alonso*, Vanesa Gallardo Arriero*, \\ laura Blázquez Rollón*, Irene Folguera Expósito*, José María Ruiz Sánchez de León**. \\ * Unidad de Formación e Investigación. Departamento de Evaluación y Calidad. Madrid Salud. Ayuntamiento de Madrid. \\ ** Departamento de Psicología Experimental, Procesos Cognitivos y Logopedia. Universidad Complutense de Madrid.
}

\section{Resumen}

El modelo UPPS de impulsividad se ha propuesto recientemente, ha sido ampliamente aplicado al abuso de sustancias y es uno de los recomendados en el contexto de investigación Research Domain Criteria, RDoC. Sin embargo, su aplicación al abuso de tecnologías de la información y la comunicación (TIC) ha sido muy limitado. En el presente trabajo se reclutó a través de Internet una muestra de $\mathrm{n}=748(67 \%$ mujeres) y se administró la versión reducida de la UPPS-P, además del MULTICAGE-TIC y el Inventario de Síntomas Prefrontales (ISP-20). Las propiedades psicométricas de la UPPS-P resultaron satisfactorias en consistencia interna $(0,87>\omega>0,75)$ y validez estructural. La impulsividad medida por la UPPS-P correlacionó con todas las escalas del MULTICAGE-TIC, aunque con un tamaño del efecto muy pequeño, y con mayor magnitud con las de síntomas de mal funcionamiento prefrontal. Las dimensiones de impulsividad más relacionadas con el abuso de las TIC fueron las de Urgencia $(0,3>r>0,2)$. Se realizó un análisis estructural de todas las variables apareciendo la impulsividad como un producto del mal funcionamiento prefrontal que predecía, a través de la Urgencia Positiva, el abuso de las TIC. La impulsividad no parece ser el núcleo central del abuso de las TIC, sino los fallos en el control superior de la conducta, de los que la impulsividad sería una consecuencia, pero no la más importante. Ello hace recomendable el diseño de intervenciones de rehabilitación cognitiva que mejoren el funcionamiento de los mecanismos de control superior de la conducta en la prevención y tratamiento del abuso de las TIC.

Palabras clave: Conducta adictiva; conducta impulsiva; corteza prefrontal; modelado de ecuaciones estructurales; adicciones comportamentales; abuso de tecnologías de la información y la comunicación.

\section{Abstract}

The UPPS model of impulsivity has recently been proposed, has been widely applied to substance abuse and is one of those recommended in the context of Research Domain Criteria, RDoC. However, its application to the abuse of information and communication technologies (ICTs) has been very limited. In the present work, a sample of $\mathrm{n}=748$ (67\% females) was recruited through the Internet, and the reduced version of the UPPS-P was administered, in addition to the MULTICAGE-TIC and the Prefrontal Symptoms Inventory (PSI-20). The psychometric properties of UPPS-P were satisfactory in terms of internal consistency $(0.87>\omega>0.75)$ and structural validity. Impulsivity measured by UPPS-P correlated with all MULTICAGE-TIC scales, although with a very small effect size, and with greater magnitude with prefrontal dysfunction symptoms. The impulsivity dimension most related to ICT abuse was Urgency $(0.3>r>0.2)$. A structural analysis of all the variables was carried out, with impulsivity appearing as a product of the prefrontal malfunction that predicted, through Positive Urgency, the abuse of ICTs. Impulsivity does not seem to be the central nucleus of ICT abuse, but rather failures in the superior control of behavior, of which impulsivity would be a consequence, but not the most important. This makes it advisable to design cognitive rehabilitation interventions that improve the functioning of superior behavior control mechanisms in the prevention and treatment of ICT abuse

Keywords: Addictive behavior; impulsive behavior; compulsive behavior; prefrontal cortex; public health; modeling of structural equations; behavioral addictions; abuse of information and communication technologies. 


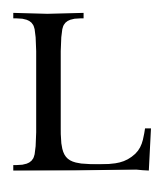

a impulsividad es un constructo psicológico ampliamente estudiado y habitualmente relacionado con diferentes manifestaciones psicológicas. Sin embargo, no existe un consenso teórico sobre el verdadero sentido del constructo, que ha sido definido de formas muy diferentes en cada enfoque teórico (Nigg, 2017). En general hace referencia a conductas emitidas sin suficiente reflexión, orientadas a metas inmediatas, sin cálculo de consecuencias a medio y largo plazo (Evenden, 1999), si bien también pueden representar, en determinadas circunstancias, opciones con valor adaptativo (Dickman, 1990). Este tipo de conductas se relaciona habitualmente con múltiples manifestaciones psicopatológicas, incluyendo conductas autolesivas y autolíticas (Lockwood, Daley, Townsend y Sayal, 2017), comportamientos violentos (Bresin, 2019) y trastornos de la personalidad (Gagnon, 2017), entre otros muchos. A partir de estudios neuropsicológicos se han ido identificando los sustratos neurales del constructo (Bari y Robbins, 2013; Chamberlain y Sahakian, 2007) que debe considerarse necesariamente multidimensional (Rochat, Billieux, Gagnon y Van der Linden, 2018).

Uno de los ámbitos en los que más se ha estudiado la impulsividad es en la adicción a sustancias, llegando a considerarse que la impulsividad es un marcador de vulnerabilidad para el desarrollo de conductas adictivas (Lee, Hoppenbrouwers y Franken, 2019; Verdejo-García, Lawrence y Clark, 2008), algo que ha sido probado en estudios con animales (Dalley, Everitt y Robbins, 2011). También hay evidencias de un incremento de la impulsividad asociado a las conductas adictivas sin sustancias (Grant y Chamberlain, 2014; Simşek, Zincir, Özen y Ceyhan, 2019), aunque aparece una gran heterogeneidad entre los diferentes estudios (Carvalho, Sette y Ferrari, 2018). Desde la consideración neuropsicológica, la inhibición de respuestas es una habilidad relacionada con la integridad de la corteza prefrontal dorsolateral que permite la interrupción o no ejecución de una conducta automatizada o un hábito adquirido cuando la no interrupción o la ejecución de la conducta va a resultar inadecuada y va a traducirse en un error (Fuster, 1997). Los déficits en el sistema de inhibición de respuestas y el control inhibitorio constituirían un elemento central en los comportamientos adictivos, según el consenso recientemente obtenido por un grupo de científicos en el marco del proyecto de investigación Research Domain Criteria, RDoC (Yücel et al., 2019).

Este mismo grupo considera que uno de los instrumentos más adecuados para su medida es el UPPS (Whiteside y Lynam, 2001). Los autores de esta prueba observaron la confusión reinante entre las diversas conceptualizaciones de la impulsividad y decidieron renunciar a un posicionamiento específico sobre la naturaleza o las causas de la impulsividad, intentando capturar lo que los autores creían que eran diversos caminos etiológicos hacia el comporta- miento impulsivo. Para ello, evaluaron mediante un análisis factorial exploratorio las diversas facetas relacionadas con la impulsividad del instrumento NEO-PI-R (Costa y McCrae, 1992) y hasta ocho escalas de impulsividad de muy diversas orientaciones teóricas. Con el conjunto de ítems seleccionados de cada uno de los factores, los autores desarrollaron el nuevo cuestionario, denominado UPPS Impulsive Behavior Scale, en el que identificaron cuatro rasgos: Urgencia negativa (conducta impulsiva que surge como reacción ante un intenso afecto negativo); [Falta de] Premeditación, que implica la habilidad para elegir una opción tomando en cuenta las posibles consecuencias; [Falta de] Perseverancia (habilidad para permanecer en las tareas, especialmente si son difíciles o aburridas); y Búsqueda de sensaciones (tendencia a buscar actividades nuevas o que suponen riesgo). Estas dimensiones permitieron comprender la impulsividad desde sus aspectos de carácter emocional/afectivo (en Urgencia y Búsqueda de sensaciones) así como desde vertientes más cognitivas (en Falta de Perseverancia y Premeditación). En la revisión de la UPPS original (UPPS-P; Lynam, Smith, Cyders, Fischer y Whiteside, 2007) incorporaron una quinta dimensión: la Urgencia positiva, definida como la propensión a actuar precipitadamente frente a un intenso afecto positivo. Estos cinco rasgos de impulsividad pueden ser medidos a través de la UPPS-P, de 59 ítems, del que se ha obtenido posteriormente una versión reducida de 20 ítems (Billieux et al., 2012).

Los rasgos propuestos por el modelo UPPS han encontrado sólida vinculación con sustratos neurales, en cierto grado específicos para cada uno de ellos, implicando prioritariamente a circuitos fronto-corticales con estructuras subcorticales (Rochat et al., 2018). Estos hallazgos se han replicado especialmente en el estudio de las conductas adictivas a diversas sustancias (Yücel et al., 2019).

La versión inicial, de cuatro subescalas, se ha utilizado con frecuencia creciente en el estudio de la adicción a sustancias (Whiteside y Lynam, 2003; Magid y Colder, 2007) y también en conductas adictivas sin sustancia (Billieux, Rochat, Rebetez y Van der Linden, 2008; Billieux, Van der Linden, M. y Rochat, 2008; Billieux et al., 2011; Rømer Thomsen et al., 2018), entre otros problemas psicológicos. También la versión revisada UPPS-P se ha utilizado en el estudio del abuso de sustancias como el alcohol (McCarty, Morris, Hatz y McCarthy, 2017), el cannabis (VanderVeen, Hershberger y Cyders, 2016) y el tabaco (Kale, Stautz y Cooper, 2018).

Por el contrario, apenas se han encontrado estudios que lo apliquen en las denominadas conductas adictivas sin sustancias. Existe actualmente una fuerte controversia sobre si tales conductas deben ser realmente consideradas como adicciones, predominando la consideración de que no debería admitirse tal asimilación (Billieux, Schimmenti, Khazaal, Maurage y Heeren, 2015; Panova y Carbonell, 2018; Yu y Sussman, 2020). En el polo opuesto, muchos autores encuentran que los circuitos implicados en las denominadas adicciones comportamentales son en esencia 
los mismos que en la adicción a sustancias (Horvath et al., 2020; Yao et al., 2017). Lo que ambas perspectivas comparten es la consideración de que en ambas se produce una hipofunción prefrontal que se traduce en una pérdida del control superior de la conducta.

La UPPS se ha aplicado en el estudio de la actividad sexual online (Savvidou et al., 2017), el uso problemático de Internet (Navas, Torres, Cándido y Perales, 2014) y el juego patológico (Jara-Rizzo et al., 2019; Wéry, Deleuze, Canale y Billieux, 2018). Mientras en el juego patológico se relaciona principalmente con la Urgencia negativa, la actividad sexual online lo hace especialmente con la Urgencia positiva y ninguna dimensión con el abuso de Internet. Estas diferencias potencialmente podrían servir para clasificar conductas que se mantienen por reforzamiento negativo o positivo.

La UPPS-P ha sido traducida y validada en español, en su versión completa (Verdejo-García, Lozano, Moya, Alcázar y Pérez-García, 2010) y en la reducida (Cándido, Orduña, Perales, Verdejo-García y Billieux, 2012). El presente trabajo tiene como objetivo estudiar algunas propiedades psicométricas de la UPPS-P reducida, para posteriormente conocer las relaciones entre las dimensiones de impulsividad del modelo UPPS, el uso/abuso de tecnologías de la información y la comunicación (TIC) y los síntomas de mal funcionamiento prefrontal.

\section{Método}

\section{Participantes}

Se obtuvo una muestra de $n=764$. No se estableció ningún criterio de exclusión, especialmente en lo relativo a la edad, pues eran de interés las respuestas en todos los grupos etarios. Tras un análisis de detección de outliers se excluyó a 16 participantes que mostraron puntuaciones atípicas $(2,1 \%)$, quedando la muestra final reducida a $n=748$.

Tabla 1. Descriptivos de la muestra.

\begin{tabular}{lccc}
\hline & Hombres & Mujeres & Total \\
\hline $\mathrm{n}$ & $245(32,8 \%)$ & $503(67,2 \%)$ & 748 \\
\hline Edad & & & \\
\hline $18-24$ & $28(23,7 \%)$ & $90(76,3 \%)$ & $118(15,8 \%)$ \\
$25-30$ & $38(36,2 \%)$ & $67(63,8 \%)$ & $105(14,0 \%)$ \\
$31-45$ & $67(33,5 \%)$ & $133(66,5 \%)$ & $200(26,7 \%)$ \\
$46-60$ & $86(35,2 \%)$ & $158(64,8 \%)$ & $244(32,6 \%)$ \\
$>60$ & $26(32,1 \%)$ & $55(67,9 \%)$ & $81(10,8 \%)$ \\
\hline Estudios & & & \\
\hline Primarios o menos & $8(44,4 \%)$ & $10(55,6 \%)$ & $18(2,4 \%)$ \\
Secundaria obligatoria & $13(72,2 \%)$ & $5(27,8 \%)$ & $18(2,4 \%)$ \\
Secundaria posobligatoria & $52(47,7 \%)$ & $57(52,3 \%)$ & $109(14,6 \%)$ \\
Estudiante universitario & $18(24,7 \%)$ & $55(75,3 \%)$ & $73(9,8 \%)$ \\
Titulado universitario & $154(29,1 \%)$ & $376(70,5 \%)$ & $530(70,9 \%)$ \\
\hline
\end{tabular}

En la Tabla 1 se presentan los descriptivos de la muestra final. El 93,6\% eran nacidos y residentes en España.

\section{Instrumentos}

UPPS-P, versión reducida de 20 ítems (Lynam, 2013), en su versión española (Cándido et al., 2012). Mide cinco rasgos de impulsividad (4 ítems cada uno): Urgencia negativa, Falta de premeditación, Falta de perseverancia, Búsqueda de sensaciones y Urgencia positiva. Los ítems se responden en una escala tipo Likert de cuatro puntos, de 1 (rotundamente de acuerdo) a 4 (rotundamente en desacuerdo). La puntuación se invierte en las dos escalas de Urgencia y en la de Búsqueda de sensaciones para que todas puedan corregirse en dirección a la impulsividad, puntuando cada una de ellas entre 4 y 16 . La consistencia interna de las 5 escalas, estimadas mediante el $\alpha$ de Cronbach, osciló entre 0,61 y 0,81 , con las dos escalas de Urgencia por debajo del 0,7 , que se tiene por límite inferior admisible.

MULTICAGE-TIC, cuestionario de 20 ítems, compuesto de 5 escalas, que interrogan sobre problemas relacionados con el uso de Internet, Móvil, Videojuegos, Mensajería Instantánea y Redes Sociales (Pedrero-Pérez et al., 2018). Está basado en el MULTICAGE CAD-4, cuestionario de cribado de conductas compulsivas, con y sin sustancias (Pedrero-Pérez et al., 2007), que ha sido utilizado en atención primaria (p. ej., Reneses et al., 2015), adicciones comportamentales (p. ej., Megías et al., 2018) y adicción a sustancias (p. ej., Navas, Torres, Cándido y Perales, 2014). Posteriormente se ha incluido una escala de uso/abuso del móvil (Rodríguez-Monje et al., 2019). En el MULTICAGE-TIC se formulan cuatro preguntas, con respuesta dicotómica (SÍ/ $\mathrm{NO}$ ), por cada conducta problema, interrogando: ítem 1, estimación de exceso en la dedicación temporal; ítem 2, estimación de otros significativos; ítem 3, dificultad para no realizar la conducta; ítem 4, dificultades en interrumpir voluntariamente la conducta. La puntuación de cada escala es el número de respuestas afirmativas, por lo que oscila entre 0 y 4 puntos, correspondiendo el 0 a inexistencia del problema y 4 a existencia de abuso. El estudio psicométrico mostró adecuada consistencia interna de todas sus escalas $(0,74<\omega<0,93)$ y evidencias de validez estructural.

Inventario de Síntomas Prefrontales, versión de cribado (ISP-20; Pedrero-Pérez, Ruiz-Sánchez de León, Morales-Alonso, Pedrero-Aguilar y Fernández-Méndez, 2015) que explora síntomas de mal funcionamiento en la vida diaria relacionados con alteraciones neuropsicológicas atribuibles a la corteza prefrontal. Se trata de una escala de 20 ítems que se responden en una escala de tipo Likert (0: nunca o casi nunca; 1 : pocas veces; 2 : a veces sí y a veces no; 3: muchas veces; 4: siempre o casi siempre). El estudio factorial halló una solución de tres factores: problemas en el control comportamental, problemas en el control emocional y problemas en la conducta social. Mayores puntuaciones se corresponden con más síntomas de mal fun- 
cionamiento de origen prefrontal. La validación tanto en población general como en personas con conductas adictivas en tratamiento informó de adecuada consistencia interna de todas las subescalas $\left(0,87<\alpha_{\mathrm{s}}<0,89\right)$. En la muestra de este estudio, la consistencia multivariada de la prueba completa fue $\alpha_{\mathrm{s}}=0,91$ y la de las escalas $0,81<\alpha_{\mathrm{s}}<0,90$.

\section{Procedimiento}

Puesto que la población diana era la de usuarios habituales de TIC, se elaboró una encuesta mediante Google Docs $^{\circledR}$ y se solicitó la participación anónima y voluntaria a través de programas de mensajería instantánea (WhattsApp $^{\circledR}$ ), redes sociales (Facebook ${ }^{\circledR}$, Instagram ${ }^{\circledR}$ ) y correo electrónico. Al tiempo, se solicitaba la difusión a los contactos de los participantes, utilizando una técnica de muestreo en cadena. Se incluyó en el cuestionario online una restricción para que no pudiera cumplimentarse más de una vez desde el mismo dispositivo. Puesto que la participación era voluntaria, se informó de los objetivos del estudio, pero no se incluyó consentimiento informado, que iba implícito en la cumplimentación de la prueba. La recogida de datos se inició el 2 de enero de 2019 y se detuvo el 12 de febrero, obteniendo finalmente una muestra de $n=764$. Esta muestra se consideró suficiente puesto que la razón entre el n muestral y el número de ítems (en total, 60) era superior a 10, como se considera habitualmente, según criterios de máxima exigencia.

\section{Análisis de datos}

En primer lugar, se realizó un análisis de detección de outliers mediante la distancia de Mahalanobis para excluir las puntuaciones atípicas, utilizando como criterio una $\mathrm{p}<$ 0,001. A continuación, se obtuvieron los descriptivos univariados de los ítems y se aplicó el criterio de Mardia (1970) para probar si los datos se ajustaban a una distribución normal multivariada. Se efectuó un análisis factorial confirmatorio, utilizando, en primer lugar, el método de máxima verosimilitud para favorecer la comparabilidad con estudios previos, y a continuación un análisis de mínimos cuadrados no ponderados, como método más adecuado a la naturaleza de los datos (Morata-Ramírez, Holgado-Tello, Barbero-García y Méndez, 2015). Se compararon dos posibles soluciones factoriales, aplicando los indicadores de ajuste proporcionados por el programa AMOS 21: absolutos (GFI, AGFI, RMR), relativos (NFI, RFI) y parsimoniosos (PGFI, PNFI). Se consideran valores adecuados los que superen el 0,95 para GFI, AGFI, NFI y RFI, los menores de 0,05 para RMR y los más próximos a 1 en PGFI y PNFI. Una vez seleccionado el mejor modelo, se configuró la estructura del cuestionario, también mediante el programa AMOS 21. La consistencia interna se estudió mediante varios estimadores, como se recomienda cuando los datos no proceden de variables lineales ni se distribuyen de acuerdo a la normalidad (Revelle y Zinbarg, 2009; Sijtsma, 2009); en concreto, se utilizaron el alfa de Cronbach estandarizado $\left(\alpha_{\mathrm{s}}\right.$; Enders y Bandalos, 1999) y el omega de McDonald $(\omega)$. Se realizó un estudio correlacional mediante la $\mathrm{r}$ de Pearson y un análisis de regresión lineal por pasos, constatando la aportación al modelo mediante la $\mathrm{R}^{2}$ y el tamaño del efecto mediante la $\beta$. En las correlaciones múltiples se aplicó la corrección de Bonferroni para evitar la comisión del Error Tipo I. Finalmente se efectuó un análisis de caminos (path analysis) para relacionar estructuralmente todas las variables previamente estudiadas y mediante el método y los estimadores de ajuste previamente utilizados. Para todos los análisis se utilizaron el paquete estadístico SPSS 22 y el programa AMOS 21, salvo para la obtención de los estimadores de consistencia interna, obtenidos mediante el programa FACTOR 10.10.01 (Lorenzo-Seva y Ferrando, 2006).

\section{Resultados}

\section{Análisis factorial confirmatorio (AFC)}

Se aplicó el criterio de Mardia que informó de que la distribución de los ítems no se ajustaba a la normalidad multivariada $(\mathrm{p}<0,001)$. Se estudió en primer lugar si el modelo teórico se ajustaba a los datos obtenidos en el presente estudio. Se efectuó en primer lugar un análisis de máxima verosimilitud que proporcionó indicadores de ajuste aceptables en casi todos los casos (CMIN/DF= 3,28; $\mathrm{NFI}=0,905 ; \mathrm{RFI}=0,887 ; \mathrm{IFI}=0,932 ; \mathrm{TLI}=0,919 ; \mathrm{CFI}=0,932 ;$ $\mathrm{PNFI}=0,760 ; \mathrm{RMSEA}=0,055)$. La mayor parte de los estudios previos utiliza este método, aquí calculado para facilitar la comparabilidad de los resultados. Sin embargo, y dada la naturaleza de los datos (escala Likert no continua y ausencia de normalidad multivariada en la distribución de los datos), se realizó a continuación un análisis de mínimos cuadrados no ponderados, como método más adecuado. Se estudiaron los indicadores de ajuste de las soluciones de 3 factores (agrupando los de Urgencia en un único factor, al igual que los de Falta de Perseverancia y Premeditación en otro) y 5 factores. Ambas soluciones mostraron un adecuado ajuste a los datos, si bien la de 5 factores (GFI= 0,985; $\mathrm{AGFI}=0,980 ; \mathrm{PGFI}=0,750 ; \mathrm{NFI}=0,973 ; \mathrm{RFI}=0,968$; $\mathrm{PNFI}=0,820 ; \mathrm{RMR}=0,028)$ se mostró ligeramente superior a la de 3 factores $(\mathrm{GFI}=0,959 ; \mathrm{AGFI}=0,949 ; \mathrm{PGFI}=0,763$; $\mathrm{NFI}=0,930 ; \mathrm{RFI}=0,920 ; \mathrm{PNFI}=0,817 ; \mathrm{RMR}=0,046 . \mathrm{En}$ la Figura 1 se muestra el modelo resultante.

\section{Consistencia interna}

En la Tabla 2 se muestran los estimadores de consistencia interna de las escalas de la UPPS-P reducido. Se puede observar que, como sucedía en el estudio de validación (Cándido et al., 2012), las dos escalas de Urgencia presentan valores inaceptables de consistencia interna $(<0,70)$ cuando se aplica el $\alpha$ de Cronbach, pero aceptables en todos los casos cuando se aplican los estimadores más apropiados a la naturaleza de los datos. 


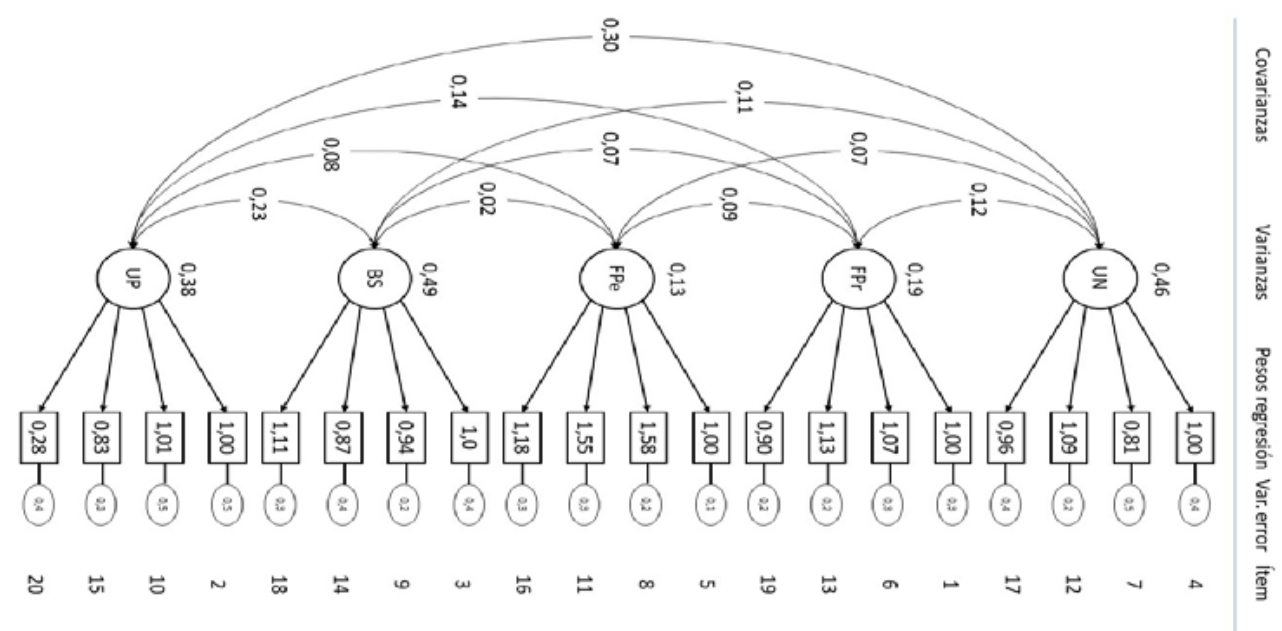

Figura 1. Estructura de la UPPS-P reducido. Nota. $\mathrm{UN}=$ Urgencia Negativa; $\mathrm{FPr}=$ Falta de Premeditación; $\mathrm{FPe}=$ Falta de Perseverancia; BS= Búsqueda de Sensaciones; UP= Urgencia Positiva.

Tabla 2. Estimadores de consistencia interna de la UPPS-P reducida.

\begin{tabular}{lccc}
\hline & $\mathbf{a}$ & as & $\boldsymbol{\omega}$ \\
\hline Urgencia Negativa & 0,67 & 0,87 & 0,87 \\
Falta de Premeditación & 0,82 & 0,82 & 0,82 \\
Falta de Perseverancia & 0,78 & 0,86 & 0,86 \\
Búsqueda de Sensaciones & 0,84 & 0,89 & 0,89 \\
Urgencia Positiva & 0,67 & 0,73 & 0,75 \\
\hline
\end{tabular}

Nota. $\mathrm{a}=$ alfa de Cronbach; as= ídem estandarizada; $\omega=$ omega de McDonald.

\section{Relación con abuso de TIC}

En la Tabla 3 se observan las correlaciones obtenidas entre las escalas de la UPPS-P y el MULTICAGE-TIC. Como puede verse, aparecen correlaciones significativas en casi todos los casos, salvo en el uso/abuso de videojuegos. Sin embargo, el tamaño del efecto de tales diferencias es muy bajo. En la Tabla 4 se muestran los modelos de regresión resultantes para cada escala del MULTICAGE-TIC. En todos los casos, la proporción de la varianza del uso/abuso de cada TIC es muy bajo, siendo las escalas de Urgencia (positiva y negativa) las que más aportan a los modelos, aunque nuevamente con un tamaño del efecto muy pobre.
Tabla 3. Correlaciones bivariadas entre las escalas de la UPPS-P reducida y el MULTICAGE-TIC.

\begin{tabular}{|c|c|c|c|c|c|}
\hline & 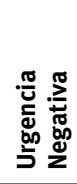 & 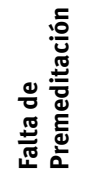 & 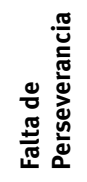 & 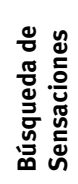 & 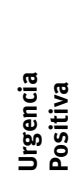 \\
\hline Internet & $0,20^{*}$ & $0,18^{*}$ & $0,14^{*}$ & $0,15^{\star}$ & $0,24^{*}$ \\
\hline Móvil & $0,22^{*}$ & $0,19^{\star}$ & $0,12^{*}$ & $0,13^{*}$ & $0,26^{*}$ \\
\hline Vídeo & 0,10 & 0,10 & 0,08 & $0,12^{*}$ & $0,15^{*}$ \\
\hline Instantánea & $0,22^{*}$ & $0,12^{\star}$ & 0,10 & 0,10 & 0,20 * \\
\hline Redes & $0,17^{*}$ & $0,18^{*}$ & $0,11^{*}$ & $0,20^{*}$ & $0,22^{*}$ \\
\hline
\end{tabular}

Nota. ${ }^{*}$ Correlación significativa tras corrección de Bonferroni (p< o,005).

\section{Relación con sintomatología prefrontal}

En la Tabla 6 se muestran las correlaciones obtenidas entre las escalas de la UPPS-P y las del ISP-20. En este caso se observa que aparecen correlaciones con considerable tamaño del efecto entre las dos escalas de Urgencia y la de Falta de perseverancia con todas las subescalas y la puntuación completa del ISP-20 y, con menor magnitud, en el resto.

Tabla 4. Modelos de regresión de las escalas de la UPPS-P reducido sobre cada una de las escalas del MULTICAGE-TIC.

\begin{tabular}{|c|c|c|c|c|c|c|}
\hline & $\begin{array}{l}\text { Urgencia } \\
\text { Negativa }\end{array}$ & $\begin{array}{c}\text { Falta de } \\
\text { Premeditación }\end{array}$ & $\begin{array}{c}\text { Falta de } \\
\text { Perseverancia }\end{array}$ & $\begin{array}{l}\text { Búsqueda de } \\
\text { Sensaciones }\end{array}$ & Urgencia Positiva & \\
\hline & \multicolumn{5}{|c|}{$R^{2 * 100(\beta)}$} & $\begin{array}{c}\text { Total \% varianza } \\
\text { explicada }\end{array}$ \\
\hline Internet & $0,4 \%(0,09)$ & $0,9 \%(0,10)$ & & & $5,5 \%(0,16)$ & $6,8 \%$ \\
\hline Móvil & $0,6 \%(0,10)$ & $0,9 \%(0,10)$ & & & $6,6 \%(0,17)$ & $8,1 \%$ \\
\hline Vídeojuegos & & & & & $2,2 \%(0,15)$ & $2,2 \%$ \\
\hline Mensajería Instantánea & $4,7 \%(0,16)$ & & & & $0,7 \%(0,11)$ & $5,4 \%$ \\
\hline Redes Sociales & & $0,9 \%(0,11)$ & & $1,3 \%(0,13)$ & $4,8 \%(0,13)$ & $7,0 \%$ \\
\hline
\end{tabular}


Tabla 5. Correlaciones bivariadas entre las escalas de la UPPS-P y el ISP-20.

\begin{tabular}{|c|c|c|c|c|c|}
\hline ISP-20 & $\begin{array}{l}\text { Urgencia } \\
\text { Negativa }\end{array}$ & $\begin{array}{c}\text { Falta } \\
\text { Premeditación }\end{array}$ & $\begin{array}{c}\text { Falta } \\
\text { Perseverancia }\end{array}$ & $\begin{array}{c}\text { Busca } \\
\text { Sensaciones }\end{array}$ & $\begin{array}{c}\text { Urgencia } \\
\text { Positiva }\end{array}$ \\
\hline Problemas en la conducta social & $0,31^{\star}$ & $0,26^{*}$ & $0,22^{\star}$ & $0,25^{\star}$ & $0,36^{\star}$ \\
\hline Problemas en el control ejecutivo & $0,40^{*}$ & $0,27^{\star}$ & $0,47^{\star}$ & $0,12^{\star}$ & $0,35^{\star}$ \\
\hline Total & $0,50^{*}$ & $0,31^{*}$ & $0,44^{*}$ & $0,18^{\star}$ & $0,45^{\star}$ \\
\hline
\end{tabular}

Nota. * Correlación significativa tras corrección de Bonferroni ( $p<0,025)$.

\section{Modelo estructural general}

En la Figura 2 se muestran las relaciones predictivas entre todas las variables utilizadas. Para simplificar la imagen se han efectuado dos restricciones: (a) se ha utilizado las cinco subescalas, propuestas por los autores como mejor solución; y (b) se han eliminado los pesos de regresión menores de 0,15. El modelo así obtenido alcanzó adecuados indicadores de ajuste (GFI=0,997; AGFI=0,992; $\mathrm{NFI}=0,972 ; \mathrm{RFI}=0,936)$, aunque mejorables en otros casos $(\mathrm{RMR}=0,479 ; \mathrm{PGFI}=0,363$; PNFI=0,424). Puede observarse como, por una parte, la mayor capacidad predictiva corresponde a la Sintomatología prefrontal sobre las subescalas de la UPPS-P, y, por otra, que la Urgencia positiva predice, aunque con bajo tamaño del efecto, todas las escalas de uso/abuso del MULTICAGE-TIC. La Urgencia negativa solo muestra una pobre capacidad predictiva sobre el uso/ abuso de Mensajería instantánea, la Falta de premeditación predice el uso/abuso del Móvily las Redes sociales, la Búsqueda de sensaciones sólo a esta última y la Falta de perseverancia no predice significativamente ninguna escala de TIC.

\section{Discusión}

El objetivo del presente estudio ha sido estudiar la aplicación del cuestionario UPPS-P, en su versión reducida de 20 ítems, en una muestra de personas que hacen uso o abuso de las tecnologías de la información y la comunicación. La prueba ha mostrado adecuadas propiedades psicométricas en su aplicación a la muestra obtenida en el presente estudio. Por una parte, el análisis factorial confirmatorio

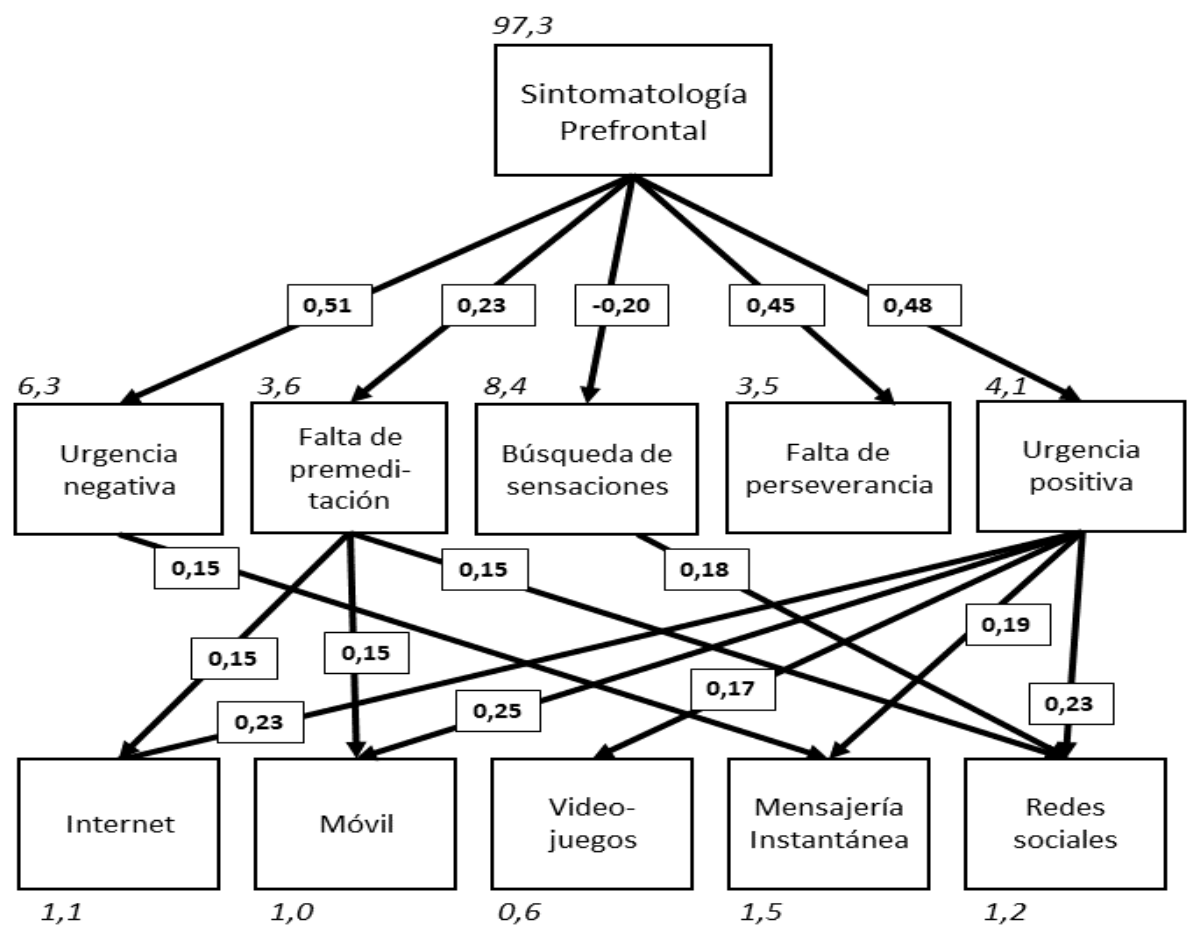

Figura 2. Modelo estructural relacionando la Sintomatología prefrontal, las subescalas de la UPPS-P y las escalas de uso/abuso de TIC.

Nota. En cursiva, varianza del error; en recuadro y negrita, pesos de regresión estandarizados. Se han omitido los pesos de regresión inferiores a $|0,15|$. 
ha obtenido adecuados indicadores de ajuste a los datos de la estructura teórica de cinco escalas. Como en el artículo de validación inicial de la versión española (Cándido et al., 2012), se ha probado una solución alternativa de tres escalas, en las que se fusionan, por una parte, las dos de Urgencia y, por otra, las de Falta de Premeditación y de Perseverancia, que también muestra un adecuado ajuste a los datos, pero mejorado por la de cinco escalas.

La consistencia interna de las cinco escalas resultó adecuada en todos los casos cuando se utilizaron estimadores multivariados. Esto no sucedió cuando en el estudio de validación se aplicó simplemente el $\alpha$ de Cronbach, algo inaceptable en el nivel de conocimiento actual (McNeish, 2018) y, sin embargo, habitual en los estudios previos de validación del cuestionario (Billieux et al., 2012; Bteich, Berbiche y Khazaal, 2017; D’Orta et al., 2015; Dugré, Giguére, Percie du Sert, Potvin y Dumais, 2019; Fossati et al., 2010; Verdejo et al., 2010).

Cuando se han puesto en relación las escalas de la UPPS-P con las del MULTICAGE-TIC se observa que casi todas las correlaciones alcanzan significación estadística, pero que el tamaño del efecto es muy pequeño en todos los casos: el máximo coeficiente de mutua determinación es el que se establece entre el uso/abuso del móvil y la $U r$ gencia positiva $\left(r^{2}=0,068\right)$, lo que se interpreta como que cada variable sólo es capaz de predecir el 6,8\% de la otra. Estos resultados contrastan con los obtenidos sobre la misma muestra cuando se exploró la compulsividad relacionada con las TIC, alcanzando algunas variables hasta el $40 \%$ de mutua determinación (Pedrero-Pérez, Morales-Alonso y Ruiz-Sánchez de León, 2021). A tenor de estos datos cabe deducir que el abuso de las TIC es una conducta que se rige mejor por las reglas de la compulsión (evitación o evasión del malestar, regida por el reforzamiento negativo) que por las de la impulsividad (búsqueda de la gratificación, regida por el reforzamiento positivo). En realidad, la Urgencia negativa definida por el modelo UPPS no difiere de la definición de compulsividad: los autores definen la Urgencia negativa como la tendencia a experimentar fuertes impulsos, frecuentemente bajo condiciones de afecto negativo, de modo que es probable que quienes puntúan alto en Urgencia negativa se involucren en conductas impulsivas con el fin de aliviar los efectos negativos a pesar de las consecuencias dañinas a largo plazo de estas acciones (Whiteside y Lynam, 2001).

Cuando se elimina la varianza común en un modelo de regresión, se observa que el conjunto de escalas de impulsividad predice, como máximo, el $8 \%$ de las escalas de abuso de TIC, y que sólo la Urgencia positiva aporta porciones significativas a los modelos, aunque no alcanza en ningún caso el 7\%. La excepción es el uso/abuso de la Mensajería instantánea, que sería mejor predicha por la Urgencia negativa. Dicho de otro modo, el uso del móvil y sus aplicaciones obedecería a la gratificación que proporcionan, mientras que el uso/abuso de la Mensajería instantánea estaría regida por la reducción del malestar que provocaría la incertidumbre de desconocer el contenido de los mensajes o como vía de escape del malestar mediante la producción de mensajes. Sin embargo, en ambos casos la aportación de las escalas de impulsividad es mínima en comparación con la que se obtiene al considerar la compulsividad (Pedrero-Pérez et al., 2021).

Cuando se correlacionan las escalas de impulsividad con las de síntomas de mal funcionamiento prefrontal aparecen relaciones consistentes con las escalas de Urgencia y con la de Falta de perseverancia, y algo menores con la Falta de premeditación. El efecto es mayor cuando se relacionan con problemas en el funcionamiento ejecutivo, como cabría esperar. Las escalas de Urgencia correlacionan fuertemente también con los problemas para el Control emocional, como también era previsible. En cambio, la Búsqueda de sensaciones alcanza un tamaño del efecto muy bajo en todas sus correlaciones. Probablemente esta última representa más un rasgo estable de personalidad (Hughson et al., 2019), mientras que el resto de escalas de la UPPS-P se aplican a tendencias de funcionamiento comportamental más dependientes del contexto estimular.

El modelo estructural conjunto relaciona los tres niveles estudiados: síntomas de mal funcionamiento prefrontal, impulsividad y uso/abuso de TIC. Lo que se puede observar es la fuerte capacidad predictiva del mal funcionamiento prefrontal sobre todos los aspectos del comportamiento impulsivo y el papel central de la Urgencia positiva sobre el abuso de las TIC. La Urgencia en la búsqueda de reforzamiento reduce la capacidad reflexiva y favorece la implicación en el uso de las TIC más allá del control prefrontal, como veíamos previamente, por el fallo de los mecanismos de control ejecutivo, pero también mediante una falta de control de los inputs emocionales. Este modelo sugiere que la mejor manera de mejorar el uso y reducir el abuso de las TIC sería el desarrollo de programas de estimulación y rehabilitación cognitiva que mejoraran los mecanismos superiores de control de la conducta, tanto en los aspectos ejecutivos como en los emocionales. La rehabilitación cognitiva ya ha mostrado su utilidad en el campo de las adicciones con o sin sustancias (Verdejo-García, Alcázar-Córcoles y Albein-Urios, 2019).

La principal limitación del presente trabajo es, sin duda, el método de obtención de la muestra. La difusión por redes sociales no permite controlar la calidad de la participación, la motivación y la sinceridad de los participantes, ni, por descontado, generalizar los resultados. La única manera de controlar, al menos globalmente, la calidad de las respuestas es obtener una muestra suficientemente grande como para que el porcentaje de respuestas inadecuadas pierda peso específico en los resultados globales. Se ha efectuado una detección de puntuaciones atípicas, de modo que se han podido eliminar las respuestas al azar o 
la cumplimentación inconsistente. Las pruebas de consistencia interna y validez estructural son también avales de una correcta cumplimentación. Sin embargo, este método de recogida de información ha ido adquiriendo un interés creciente y su uso se considera habitual en la investigación psicosociológica (Geisen y Murphy, 2020). Futuros estudios deben buscar métodos de muestreo que permitan generalizar los resultados.

En conclusión, la UPPS-P en su versión reducida de 20 ítems, es una prueba consistente y estructuralmente válida para explorar la impulsividad, según el modelo multidimensional UPPS. A tenor de los resultados obtenidos, los componentes impulsivos del abuso de las TIC no son el elemento central del problema, a diferencia de cuando se han estudiado los componentes compulsivos. Esta consideración puede guiar el diseño de intervenciones más eficaces que, probablemente, deberían orientarse a la mejora de los mecanismos de control cortical, ejecutivo y emocional, y a la capacidad para generar alternativas de respuesta válidas, más que al mero bloqueo o la modificación de las conductas de uso excesivo.

\section{Conflicto de intereses}

Los autores declaran que no presentan ningún conflicto de intereses.

\section{Referencias}

Bari, A. y Robbins, T. W. (2013). Inhibition and impulsivity: Behavioral and neural basis of response control. Progress in Neurobiology, 108, 44-79. doi:10.1016/j.pneurobio.2013.06.005.

Billieux, J., Chanal, J., Khazaal, Y., Rochat, L., Gay, P., Zullino, D. y Van der Linden, M. (2011). Psychological predictors of problematic involvement in massively multiplayer online role-playing games: Illustration in a sample of male cybercafé players. Psychopathology, 44, 165171. doi:10.1159/000322525.

Billieux, J., Rochat, L., Ceschi, G., Carré, A., Offerlin-Meyer, I., Defeldre, A. C.,... Van der Linden, M. (2012). Validation of a short French version of the UPPS-P Impulsive Behaviour Scale. Comprehensive Psychiatry, 53, 609-615. doi:10.1016/j.comppsych.2011.09.001.

Billieux, J., Rochat, L., Rebetez, M. M. L. y Van der Linden, M. (2008). Are all facets of impulsivity related to self-reported compulsive buying behavior? Personality and Individual Differences, 44, 1432-1442. doi:10.1016/j. paid.2007.12.011.

Billieux, J., Schimmenti, A., Khazaal, Y., Maurage, P. y Heeren, A. (2015). Are we overpathologizing everyday life? A tenable blueprint for behavioral addiction research. Journal of Behavioral Addictions, 4, 119-123. doi:10.1556/2006.4.2015.009.
Billieux, J., Van der Linden, M. y Rochat, L. (2008). The role of impulsivity in actual and problematic use of the mobile phone. Applied Cognitive Psychology, 22, 11951210. doi:10.1002/acp.1429.

Bowling, A. (2005). Mode of questionnaire administration can have serious effects on data quality. Journal of Public Health, 27, 281-291. doi:10.1093/pubmed/fdi031.

Bresin, K. (2019). Impulsivity and aggression: A meta-analysis using the UPPS model of impulsivity. $A g$ gression and Violent Behavior, 48, 124-140. doi:10.1016/j. avb.2019.08.003.

Bteich, G., Berbiche, D. y Khazaal, Y. (2017). Validation of the short Arabic UPPS-P impulsive behavior scale. $B M C$ Psychiatry, 17, 244. doi:10.1186/s12888-017-1407-y.

Cándido, A., Orduña, E., Perales, J. C., Verdejo-García, A. y Billieux, J. (2012). Validation of a short Spanish version of the UPPS-P impulsive behaviour scale. Trastornos Adictivos, 14, 73-78. doi:10.1016/S1575-0973(12)70048-X.

Carvalho, L. F., Sette, C. P. y Ferrari, B. L. (2018). Problematic smartphone use relationship with pathological personality traits: Systematic review and meta-analysis. Cyberpsychology, 12, 5. doi:10.5817/CP2018-3-5.

Chamberlain, S. R. y Sahakian, B. J. (2007). The neuropsychiatry of impulsivity. Current Opinion in Psychiatry, 20, 255-261. doi:10.1097/YCO.0b013e3280ba4989.

Costa, P. T. y McCrae, R. R. (1992). Revised NEO Personality Inventory (NEO-PI-R) and NEO Five-Factor Inventory (NEOFFI) profesional manual. Odessa, FL: Psychological Assessment Resources.

Dalley, J. W., Everitt, B. J. y Robbins, T. W. (2011). Impulsivity, compulsivity, and top-down cognitive control. Neuron, 69, 680-694. doi:10.1016/j.neuron.2011.01.020.

Dickman, S. J. (1990). Functional and dysfunctional impulsivity: Personality and cognitive correlates. Journal of Personality and Social Psychology, 58, 95-102. doi:10.1037/00223514.58.1.95

D’Orta, I., Burnay, J., Aiello, D., Niolu, C., Siracusano, A., Timpanaro, L.,... Billieux, J. (2015). Development and validation of a short Italian UPPS-P Impulsive Behavior Scale. Addictive Behaviors Reports, 2, 19-22. doi:10.1016/j. abrep.2015.04.003.

Dugré, J. R., Giguére, C. É., Percie du Sert, O., Potvin, S. y Dumais, A. (2019). The psychometric properties of a short UPPS-P Impulsive Behavior Scale among psychiatric patients evaluated in an emergency setting. Frontiers in Psychiatry, 10, 139. doi:10.3389/fpsyt.2019.00139.

Enders, C. K. y Bandalos, D. L. (1999). The effects of heterogeneous item distributions on reliability. Applied Measurement in Education, 12, 133-150.

Evenden, J. L. (1999). Varieties of impulsivity. Psychopharmacology, 146, 348-361. doi:10.1007/PL00005481.

Fossati, A., Somma, A., Karyadi, K. A., Cyders, M. A., Bortolla, R. y Borroni, S. (2016). Reliability and validity of the Italian translation of the UPPS-P Impulsive Behavior 
Scale in a sample of consecutively admitted psychotherapy patients. Personality and Individual Differences, 91, 1-6. doi:10.1016/j.paid.2015.11.020.

Fuster, J. M. (1997). The prefrontal cortex. $3^{\mathrm{a}}$ ed. New York: Raven Press.

Gagnon, J. (2017). Defining borderline personality disorder impulsivity: Review of neuropsychological data and challenges that face researchers. Journal of Psychiatry and Psychiatric Disorders, 1, 154-176. doi:10.26502/jppd.2572519X0015.

Geisen, E. y Murphy, J. (2020). A compendium of web and mobile survey pretesting methods. En P. Beatty, D. Collins, L. Kaye, J. L. Padilla, G. Willis y A. Wilmot (Eds.), Advances in questionnaire design, development, evaluation and testing (pp. 287-314). New York: John Wiley \& Sons.

Grant, J. E. y Chamberlain, S. R. (2014). Impulsive action and impulsive choice across substance and behavioral addictions: Cause or consequence? Addictive Behaviors, 39, 1632-1639. doi:10.1016/j.addbeh.2014.04.022.

Horvath, J., Mundinger, C., Schmitgen, M. M., Wolf, N. D., Sambataro, F., Hirjak, D.,... Wolf, R. C. (2020). Structural and functional correlates of smartphone addiction. Addictive Behaviors, 105, 106334. doi:10.1016/j.addbeh.2020.106334.

Hughson, A. R., Horvath, A. P., Holl, K., Palmer, A. A., Woods, L. C. S., Robinson, T. E. y Flagel, S. B. (2019). Incentive salience attribution, "sensation-seeking" and "novelty-seeking" are independent traits in a large sample of male and female heterogeneous stock rats. Scientific Reports, 9, 2351. doi:10.1038/s41598-019-39519-1.

Jara-Rizzo, M. F., Navas, J. F., Steward, T., López-Gómez, M., Jiménez-Murcia, S., Fernández-Aranda, F. y Perales, J. C. (2019). Impulsivity and problem awareness predict therapy compliance and dropout from treatment for gambling disorder. Adicciones, 31, 147-159. doi:10.20882/ adicciones. 1041 .

Kale, D., Stautz, K. y Cooper, A. (2018). Impulsivity related personality traits and cigarette smoking in adults: A meta-analysis using the UPPS-P model of impulsivity and reward sensitivity. Drug and Alcohol Dependence, 185, 149167. doi:10.1016/j.drugalcdep.2018.01.003.

Lee, R. S., Hoppenbrouwers, S. y Franken, I. (2019). A systematic meta-review of impulsivity and compulsivity in addictive behaviors. Neuropsychology Review, 29, 14-26. doi:10.1007/s11065-019-09402-x.

Lockwood, J., Daley, D., Townsend, E. y Sayal, K. (2017). Impulsivity and self-harm in adolescence: A systematic review. European Child E Adolescent Psychiatry, 26, 387402. doi:10.1007/s00787-016-0915-5.

Lorenzo-Seva, U. y Ferrando, P. J. (2006). FACTOR: A computer program to fit the exploratory factor analysis model. Behavior Research Methods, 38, 88-91. doi:10.3758/ BF03192753.
Lynam, D. R. (2013). Development of a short form of the UPPS-P Impulsive Behavior Scale. Informe técnico no publicado. 2013.

Lynam, D. R., Smith, G. T., Cyders, M. A., Fischer, S. y Whiteside, S. P. (2007). The UPPS-P questionnaire measure of five dispositions to rash action. Informe técnico no publicado. Purdue University, West Lafayette, IN.

Magid, V. y Colder, C. R. (2007). The UPPS Impulsive Behavior Scale: Factor structure and associations with college drinking. Personality and Individual Differences, 43, 1927-1937. doi:10.1016/j.paid.2007.06.013.

Mardia, K. V. (1970). Measures of multivariate skewnees and kurtosis with applications. Biometrika, 57, 519-530. doi: $10.2307 / 2334770$.

McCarty, K. N., Morris, D. H., Hatz, L. E. y McCarthy, D. M. (2017). Differential associations of UPPS-P impulsivity traits with alcohol problems. Journal of Studies on Alcohol and Drugs, 78, 617-622. doi:10.15288/jsad.2017.78.617.

McNeish, D. (2018). Thanks coefficient alpha, we'll take it from here. Psychological Methods, 23, 412-433. doi:10.1037/met0000144.

Megías, A., Navas, J. F., Perandrés-Gómez, A., Maldonado, A., Catena, A. y Perales, J. C. (2018). Electroencephalographic evidence of abnormal anticipatory uncertainty processing in gambling disorder patients. Journal of Gambling Studies, 34, 321-338. doi:10.1007/s10899-0179693-3.

Morata-Ramírez, M., Holgado-Tello, F. P., Barbero-García, I. y Mendez, G. (2015). Análisis factorial confirmatorio: Recomendaciones sobre mínimos cuadrados no ponderados en función del error Tipo I de Ji-Cuadrado y RMSEA. Acción Psicológica, 12, 79-90. doi:10.5944/ ap.12.1.14362.

Navas, J. F., Torres, A., Cándido, A. y Perales, J. C. (2014). ¿'Nada' o ‘un poco'? ¿’Mucho' o ‘demasiado'? La impulsividad como marcador de gravedad en niveles problemático y no problemático de uso de alcohol e Internet. Adicciones, 26, 146-158. doi:10.20882/adicciones.19.

Nigg, J. T. (2017). Annual research review: On the relations among self-regulation, self-control, executive functioning, effortful control, cognitive control, impulsivity, risk-taking, and inhibition for developmental psychopathology. Journal of Child Psychology and Psychiatry, 58, 361383. doi:10.1111/jcpp.12675.

Panova, T. y Carbonell, X. (2018). Is smartphone addiction really an addiction? Journal of Behavioral Addictions, 7, 252-259. doi:10.1556/2006.7.2018.49.

Pedrero-Pérez, E. J. (2010). Detección de adicciones comportamentales en adictos a sustancias en tratamiento. Trastornos Adictivos, 12, 13-18. doi:10.1016/S15750973(10) 70005-2.

Pedrero-Pérez, E. J., Morales-Alonso, S. y Ruiz-Sánchez de León, J. M. (2021). Obsesión y compulsión en el uso/ 
abuso del móvil: El OCDUS-TIC. Adicciones, 33, 149-160. doi:10.20882/adicciones.1320.

Pedrero-Pérez, E. J., Rodríguez-Monje, M. T., Gallardo-Alonso, F., Fernández-Girón, M., Pérez-López, M. y Chicharro-Romero, J. (2007). Validación de un instrumento para la detección de trastornos de control de impulsos y adicciones: El MULTICAGE CAD-4. Trastornos Adictivos, 9, 269-278. doi:10.1016/S15750973(07) 75656-8.

Pedrero-Pérez, E. J., Ruiz-Sánchez de León, J. M., Morales-Alonso, S., Pedrero-Aguilar, J. y Fernández-Méndez, L. M. (2015). Sintomatología prefrontal en la vida diaria: Evaluación de cribado mediante el inventario de síntomas prefrontales abreviado (ISP-20). Revista de Neurología, 60, 385-393. doi:10.33588/rn.6009.2014545.

Pedrero-Pérez, E. J., Ruiz-Sánchez de León, J. M., Rojo-Mota, G., Llanero-Luque, M., Pedrero-Aguilar, J., Morales-Alonso, S. y Puerta-García, C. (2018). Tecnologías de la Información y la Comunicación (TIC): Uso problemático de internet, videojuegos, teléfonos móviles, mensajería instantánea y redes sociales mediante el MULTICAGE-TIC. Adicciones, 30, 19-32. doi:10.20882/ adicciones.806.

Pedrero-Pérez, E. J., Ruiz Sánchez de León, J. M., Rojo Mota, G., Morales Alonso, S., Pedrero Aguilar, J., Lorenzo Luque, I. y González Sánchez, Á. (2016). Inventario de Síntomas Prefrontales (ISP): Validez ecológica y convergencia con medidas neuropsicológicas. Revista de Neurología, 63, 241-251. doi:10.33588/rn.6306.2016143.

Reneses, B., Garrido, S., Navalón, A., Martín, O., Ramos, I., Fuentes, M.,... López-Ibor, J. J. (2015). Psychiatric morbidity and predisposing factors in a primary care population in Madrid. International Journal of Social Psychiatry, 61, 275-286. doi:10.1177/0020764014542815.

Revelle, W. y Zinbarg, R. E. (2009). Coefficients alpha, beta, omega, and the glb: Comments on Sijtsma. Psychometrika, 74, 145. doi:10.1007/s11336-008-9102-z.

Rochat, L., Billieux, J., Gagnon, J. y Van der Linden, M. (2018). A multifactorial and integrative approach to impulsivity in neuropsychology: Insights from the UPPS model of impulsivity. Journal of Clinical and Experimental Neuropsychology, 40, 45-61.

Rodríguez-Monje, M. T., Pedrero-Pérez, E. J. P., Fernández-Girón, M., Gallardo-Alonso, F. y Sanz-Cuesta, T. (2009). Detección precoz de conductas adictivas en atención primaria: Utilización del MULTICAGE CAD-4. Atención Primaria, 41, 25-32. doi:10.1016/j. aprim.2008.04.004.

Rodriguez-Monje, M. T., Pedrero-Pérez, E. J., Rodríguez-Alonso, E., Fernández-Girón, M., Pastor-Ramos, V., Mateo-Madurga, A.,... Escriva-Ferrairo, R. (2019). MULTICAGE CAD-4 for behavioral addiction screening: Structural validity after inclusion of a scale on smartpho- ne abuse. Anales de Psicología, 35, 41-46. doi:10.6018/ analesps.35.1.324311.

Rømer Thomsen, K., Callesen, M. B., Hesse, M., Kvamme, T. L., Pedersen, M. M., Pedersen, M. U. y Voon, V. (2018). Impulsivity traits and addiction-related behaviors in youth. Journal of Behavioral Addictions, 7, 317-330. doi:10.1556/2006.7.2018.22.

Ruiz-Sánchez de León, J. M., Pedrero-Pérez, E. J., Gálvez, S., Fernández-Méndez, L. M. y Lozoya-Delgado, P. (2015). Utilidad clínica y propiedades psicométricas del inventario de síntomas prefrontales (ISP) en el daño cerebral adquirido y las demencias degenerativas. Revista de Neurología, 61, 387-394. doi:10.33588/rn.6109.2015252.

Savvidou, L. G., Fagundo, A. B., Fernández-Aranda, F., Granero, R., Claes, L., Mallorquí-Baqué, N.,... del Pino-Gutiérrez, A. (2017). Is gambling disorder associated with impulsivity traits measured by the UPPS-P and is this association moderated by sex and age? Comprehensive Psychiatry, 72, 106-113. doi:10.1016/j.comppsych.2016.10.005.

Sijtsma, K. (2009). On the use, the misuse, and the very limited usefulness of Cronbach's alpha. Psychometrika, 74, 107. doi:10.1007/s11336-008-9101-0.

Şimşek, N., Zincir, H., Özen, B. y Ceyhan, Ö. (2019). The association between Internet addiction and impulsivity among academicians. Addicta, 6, 269-281. doi:10.15805/ addicta.2019.6.2.0012.

VanderVeen, J. D., Hershberger, A. R. y Cyders, M. A. (2016). UPPS-P model impulsivity and marijuana use behaviors in adolescents: A meta-analysis. Drug and Alcohol Dependence, 168, 181-190. doi:10.1016/j.drugalcdep.2016.09.016.

Verdejo-García, A., Alcázar-Córcoles, M. A. y Albein-Urios, N. (2019). Neuropsychological interventions for decision-making in addiction: A systematic review. Neuropsychology Review, 29, 79-92. doi:10.1007/s11065-018-9384-6.

Verdejo-García, A., Lawrence, A. J. y Clark, L. (2008). Impulsivity as a vulnerability marker for substance-use disorders: Review of findings from high-risk research, problem gamblers and genetic association studies. Neuroscience E B Biobehavioral Reviews, 32, 777-810. doi:10.1016/j.neubiorev.2007.11.003.

Verdejo-García, A., Lozano, Ó., Moya, M., Alcázar, M. A. y Pérez-García, M. (2010). Psychometric properties of a Spanish version of the UPPS-P Impulsive Behavior Scale: Reliability, validity and association with trait and cognitive impulsivity. Journal of Personality Assessment, 92, 70-77. doi:10.1080/00223890903382369.

Wéry, A., Deleuze, J., Canale, N. y Billieux, J. (2018). Emotionally laden impulsivity interacts with affect in predicting addictive use of online sexual activity in men. Comprehensive Psychiatry, 80, 192-201. doi:10.1016/j.comppsych.2017.10.004.

Whiteside, S. P. y Lynam, D. R. (2001). The five factor model and impulsivity: Using a structural model of 
personality to understand impulsivity. Personality and Individual Differences, 30, 669-689. doi:10.1016/S01918869(00)00064-7.

Whiteside, S. P. y Lynam, D. R. (2003). Understanding the role of impulsivity and externalizing psychopathology in alcohol abuse: Application of the UPPS impulsive behavior scale. Experimental and Clinical Psychopharmacology, 11, 210-217. doi:10.1037/1949-2715.S.1.69.

Yao, Y. W., Liu, L., Ma, S. S., Shi, X. H., Zhou, N., Zhang, J. T. y Potenza, M. N. (2017). Functional and structural neural alterations in Internet gaming disorder: A systematic review and meta-analysis. Neuroscience $\mathcal{E}$ Biobehavioral Reviews, 83, 313-324. doi:10.1016/j.neubiorev.2017.10.029.
Yu, S. y Sussman, S. (2020). Does smartphone addiction fall on a continuum of addictive behaviors? International Journal of Environmental Research and Public Health, 17, 422. doi:10.3390/ijerph17020422.

Yücel, M., Oldenhof, E., Ahmed, S. H., Belin, D., Billieux, J., Bowden-Jones, H.,... Daglish, M. (2019). A transdiagnostic dimensional approach towards a neuropsychological assessment for addiction: An international Delphi consensus study. Addiction, 114, 1095-1109. doi:10.1111/ add.14424. 